\title{
Le plan en relief du canal de Suez de Charles Muret (1878, actualisé en 1900)
}

The relief map of the Suez Canal by Charles Muret (1878, updated in 1900)

\section{Isabelle Warmoes}

\section{OpenEdition}

Journals

Édition électronique

URL : http://journals.openedition.org/artefact/3511

DOI : 10.4000/artefact.3511

ISSN : 2606-9245

Éditeur :

Association Artefact. Techniques histoire et sciences humaines, Presses universitaires du Midi

Édition imprimée

Date de publication : 15 mars 2019

Pagination : 285-293

ISBN : 978-2-8107-0623-5

ISSN : 2273-0753

Référence électronique

Isabelle Warmoes, «Le plan en relief du canal de Suez de Charles Muret (1878, actualisé en 1900) » Artefact [En ligne], 9 | 2018, mis en ligne le 04 mars 2020, consulté le 27 novembre 2020. URL : http:// journals.openedition.org/artefact/3511 ; DOI : https://doi.org/10.4000/artefact.3511

\section{(c) (i) (9)}

Artefact, Techniques, histoire et sciences humaines est mise à disposition selon les termes de la Licence Creative Commons Attribution - Pas d'Utilisation Commerciale - Pas de Modification 4.0 International. 


\section{Le plan en relief du canal de Suez de Charles Muret (1878, actualisé en 1900)}

\section{Isabelle Warmoes}

\section{Résumé}

Le plan-relief du canal de Suez, entré dans les collections du musée des PlansReliefs en 1910, a été réalisé par Charles Muret à la demande de la Compagnie universelle du canal maritime de Suez pour l'Exposition universelle de Paris de 1878, puis largement actualisé pour celle de 1900. Destiné à rendre compte de l'importance et de l'évolution des aménagements réalisés depuis l'ouverture de cette voie commerciale, il constitue un précieux témoignage de l'état du canal de Suez, des villes nouvelles et des équipements aménagés à ses abords à la fin du xixe siècle.

\section{Mots-clés}

canal de Suez, Égypte, Charles Muret, plan-relief, xIx siècle

99 Isabelle Warmoes, « Le plan en relief du canal de Suez de Charles Muret (1878, actualisé en 1900) », Artefact, 9, 2018, p. 285-293. 


\section{The relief map of the Suez Canal by Charles Muret (1878, updated in 1900)}

\section{Abstract}

The relief map of the Suez Canal, entered into the collections of the Musée des Plans-Reliefs in 1910, was made by Charles Muret at the request of the Universal Suez Canal Company for the Paris International Exhibition of 1878, then extensively updated for that of 1900 . Intended to reflect the importance and evolution of developments since the opening of this trade route, it is now a valuable testimony to the state of the Suez Canal, new cities and equipment built around it at the end of the 19 th century.

\section{Keywords}

Suez Canal, Egypt, Charles Muret, Relief map, 19th century 


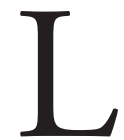

'exposition "L'épopée du canal de Suez ", présentée du 28 mars au 5 août 2018 à l'Institut du monde arabe, puis dans une version remaniée au musée d'Histoire de Marseille du 19 octobre 2018 au 31 mars $2019^{1}$, a été l'occasion d'exposer le plan en relief du canal de Suez, conservé dans les collections du musée des Plans-Reliefs (Fig. XIII, cahier couleur). Composée de quatre tables et réalisée à l'échelle du $1 / 22000$, cette œuvre spectaculaire mesure $855 \mathrm{~cm}$ de long sur $123 \mathrm{~cm}$ de large et $26,5 \mathrm{~cm}$ de haut en son point culminant. Sur une structure de panneaux de bois peints, les reliefs sont réalisés en plâtre teinté et en papier mâché agglutiné. Du sable mélangé au plâtre restitue la couleur et la topographie des sites de part et d'autre du canal. La végétation est en soie; du métal et des pierres brillantes de couleur ont aussi été utilisés.

Le plan-relief du canal de Suez, entré dans les collections du musée des Plans-Reliefs en 1910 par don du conseil d'administration de la Compagnie universelle du canal maritime de Suez, n'était plus visible du public depuis 1943, date du transfert des collections du musée hors de Paris pour les mettre à l'abri pendant la Seconde Guerre mondiale. Il était depuis conservé en réserve et avait connu divers dommages et réparations grossières. Sa présentation nécessitait la mise en œuvre d'une campagne de restauration préalable, confiée en décembre 2017 à un groupement de restauratrices dirigé par Anne Courcelle, restauratrice de sculptures. L'œuvre comportait de nombreuses fissures, des lacunes et de lourds enduits de reprises en bordure de chacune des tables, l'ensemble témoignant de remaniements successifs. Pour documenter au mieux la restauration programmée, des recherches en archives ont été menées afin de mieux dater l'œuvre et de comprendre la nature des éléments représentés. Les principaux fonds consultés ont été ceux des archives privées de la Compagnie universelle du canal maritime de Suez, déposées aux Archives nationales du monde du travail. Cette recherche a permis d'identifier précisément, parmi les diverses maquettes réalisées à la demande de la Compagnie, le plan-relief qui fait l'objet de cette étude et de documenter les différentes étapes de sa réalisation.

Depuis 1869, année d'inauguration du canal de Suez, jusqu'en 1900, la Compagnie universelle du canal maritime de Suez a fait réaliser trois

1. Gilles Gauthier (dir.), L'épopée du canal de Suez, Paris/Marseille, Gallimard/Institut du monde arabe/Musée d'Histoire de Marseille, 2018. 
plans-reliefs du canal ${ }^{2}$, commandés à chaque fois dans la perspective de leur présentation lors des expositions universelles organisées à Paris ${ }^{3}$. Le premier, réalisé en 1869 par M. Muret à l'échelle de 1/100 000, mesurait $165 \mathrm{~cm}$ de longueur sur $45 \mathrm{~cm}$ de largeur. Il est décrit comme une plaquette qui se fixe au mur, documentant le canal à l'époque de son inauguration. Un deuxième plan a été commandé en 1878 et dressé par MM. Muret et Hallé à l'échelle de 1/22 000 environ; il mesure $855 \mathrm{~cm}$ de longueur sur $123 \mathrm{~cm}$ de largeur, était posé sur un bâti en menuiserie et recouvert d'une vitrine. Un troisième a été réalisé pour l'Exposition universelle de 1889 par M. Hallé, à l'échelle du 1/20 000 environ. Il mesurait $947 \mathrm{~cm}$ de longueur sur $147 \mathrm{~cm}$ de largeur Ce plan était équipé d'un système d'éclairage permettant de restituer les équipements installés le long du canal nécessaires à la circulation des navires de nuit.

\section{Le plan en relief commandé en 1878 à Charles Muret et Hallé}

Le plan-relief conservé dans les collections du musée des Plans-Reliefs est celui réalisé en 1878. Il a été commandé par la Compagnie universelle du canal maritime de Suez pour être présenté dans le pavillon égyptien du Trocadéro lors de l'Exposition universelle de Paris de 1878. Charles Muret, géomètre de la Ville de Paris et constructeur géographe, en a assuré la fabrication, secondé par M. Hallé, décorateur à Paris. Charles Muret (1839$1921)^{4}$, dessinateur, cartographe, mathématicien, membre de la société de géographie, est admis comme géomètre de la Ville de Paris en 1857, puis promu géomètre en chef en 1871, avant d'être nommé professeur de dessin et de topographie à l'Institut d'agronomie, de 1890 à 1911. Élève et principal collaborateur de Libre Bardin (1794-1867), professeur de topographie à l'école Polytechnique, il est l'auteur de plusieurs plans-reliefs,

2. La réalisation d'autres plans-reliefs du canal a été commandée au cours du premier tiers du $\mathrm{Xx}^{e}$ siècle. Certaines villes aménagées le long du canal, comme Ismaïlia, ont aussi été représentées en plans-reliefs.

3. Plans en relief du canal. Note du 25 mai 1899. Roubaix, Archives nationales du monde du travail (ANMT), fonds " Compagnie universelle du canal maritime de Suez, puis Compagnie financière de Suez ", Carton 1995060 4153, Plans en relief du canal et des villes (1889-1908), pochette : Exposition universelle 1900.

4. Georges Charon, "Charles Muret (1839-1921), professeur de dessin graphique et de topographie» [notice nécrologique], Annales de l'Institut national agronomique, t. 16, 1922, p. 5-10. 
parmi lesquels on citera le plan-relief avec courbes de niveaux de la ville de Paris, un plan-relief de la Manche, un plan-relief d'Athènes et du Pirée, un plan-relief de Jérusalem et ses environs, un plan-relief du canal interocéanique de Panama et plusieurs plans-reliefs du canal de Suez.

À travers le plan-relief de 1878, la Compagnie universelle du canal maritime de Suez a voulu exposer au public le canal des Deux-Mers achevé et signaler les résultats de son exploitation depuis l'année de son inauguration - 1869 - jusqu'à la fin de l'année 1877. Le visiteur pouvait ainsi suivre, de station en station, de la mer Méditerranée à la mer Rouge, la voie maritime ouverte au commerce international et se rendre compte de l'importance des travaux exécutés ${ }^{5}$ :

- la ville neuve de Port-Saïd (Fig. XIV, cahier couleur), au tracé orthogonal, établie dans une zone marécageuse entre le littoral méditerranéen et le lac Menzaleh. La ville, divers bassins, entrepôts et ateliers, ont été créés pour accueillir le matériel venu d'Europe. Deux longues jetées ont été aménagées dans la mer pour créer un avant-port offrant un abri sûr aux navires. À proximité de la ville se trouvait le village des ouvriers égyptiens ;

- à mi-chemin entre les deux extrémités du canal, à proximité du lac Timsah, est fondée en plein désert la ville neuve d'Ismaïlia (Fig. XV, cahier couleur), destinée à accueillir les services administratifs et les résidences du personnel de la direction de la Compagnie du canal de Suez. La ville est alimentée par un canal d'eau douce apportant les eaux du Nil ;

- plus loin se développent les bassins des lacs Amer;

- l'agglomération de Suez, petit port de pêche, est la seule localité préexistante à la création du canal. Plutôt que de développer Suez, la Compagnie du canal a préféré créer plus à l'ouest une nouvelle ville débouchant sur la mer Rouge : Port-Tewfic (Fig. XVI, cahier couleur).

De nombreux bateaux ont été mis en place sur la maquette, reproduisant les différents types de navires empruntant le canal. Un nombre important a disparu et on en conserve la mémoire grâce aux traces d'arrachements visibles sur le plan-relief, le long du canal et à son embouchure en Méditerranée et en Mer rouge.

5. Dossier : Exposition 1878. Salle du canal de Suez (génie civil). ANMT, fonds " Compagnie universelle du canal maritime de Suez, puis Compagnie financière de Suez », Carton 1995060 1723, Expositions diverses (1878-1892). 
Ce plan en relief du canal de Suez, réalisé en 1878, a par la suite été présenté lors de l'Exposition universelle de Paris en 1889, dans le Pavillon de Suez du Champs-de-Mars. Une vue éloignée, photographie réalisée par l'ingénieur et photographe Albert Fernique (1841-1898), publiée dans la Notice sur la participation de la Compagnie. Exposition universelle de Paris $(1889)^{6}$, reproduit le plan-relief tel qu'il était exposé dans la salle principale du pavillon du Champs de Mars (Fig. 1). Dans une salle attenante, plongée dans la pénombre, était exposé le plan-relief éclairé réalisé en 1889 par M. Hallé.

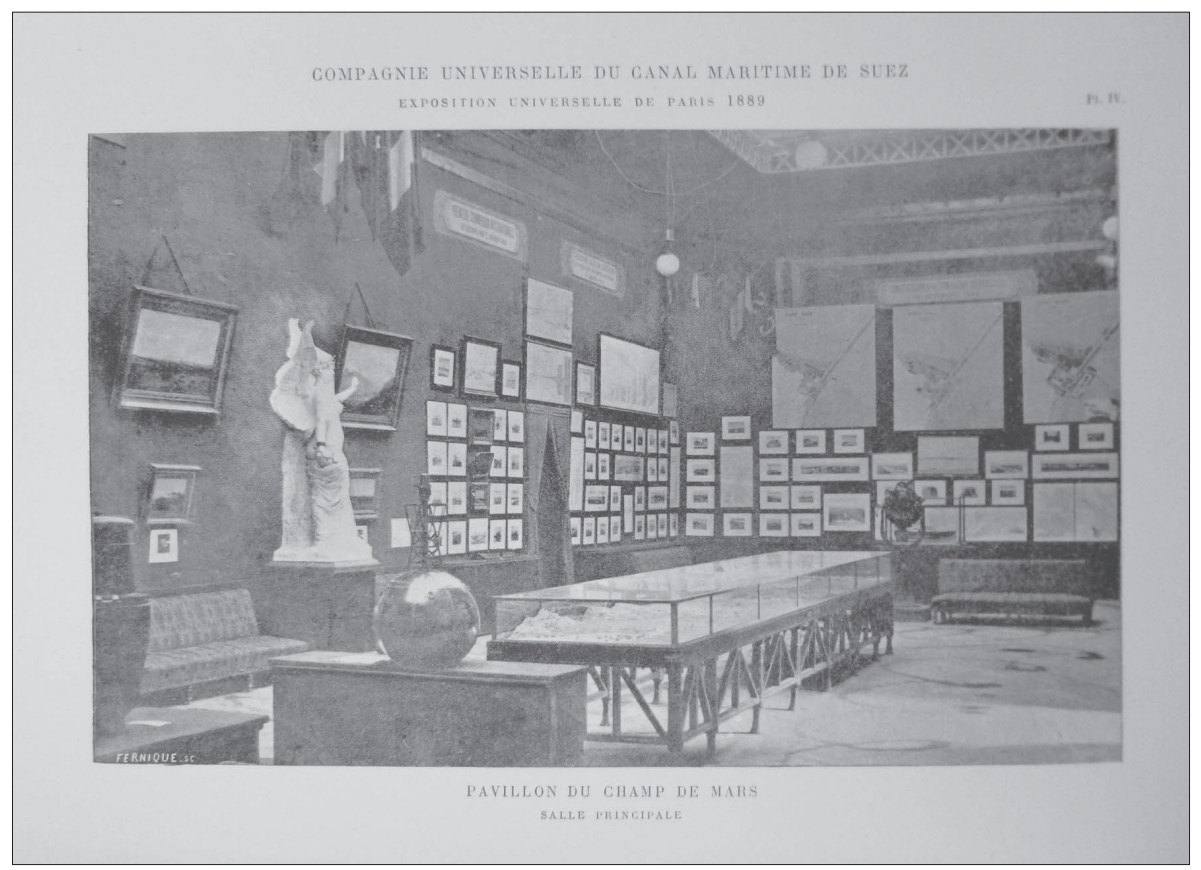

Fig. 1 - Plan-relief du canal de Suez, construit en 1878 par M. Muret et M. Hallé et exposé dans la salle principale du Pavillon de Suez du Champs de Mars lors de l'Exposition universelle de 1889

Photographie d'Albert Fernique extraite de Exposition universelle de Paris (1889). Notice sur la participation de la Compagnie, Paris, Imprimerie de la Compagnie, 1890, Pl. IV.

Cliché Isabelle Warmoes

6. Compagnie universelle du canal maritime de Suez, Exposition universelle de Paris (1889). Notice sur la participation de la Compagnie, Paris, Imprimerie de la Compagnie, 1890, pl. IV. 


\section{L'actualisation du plan en relief pour l'Exposition universelle de $\mathbf{1 9 0 0}$}

Le plan en relief réalisé en 1878 par Charles Muret et Hallé a été largement actualisé à la demande de la Compagnie du canal de Suez, pour être présenté lors de l'Exposition universelle de $1900^{7}$. C'est cet état que présente la maquette aujourd'hui. Il s'agissait pour la Compagnie d'y faire figurer les nombreuses modifications et installations servant à l'exploitation du canal qui avaient été mises en œuvre depuis 1885. Pour faire face au nombre croissant de navires et à l'augmentation de leur tonnage, une commission internationale avait été chargée en 1884 de décider des améliorations à apporter au canal. Il fut ainsi décidé de l'élargir : la largeur au plafond fut portée de 22 à $37 \mathrm{~m}$ et la profondeur de $8 \mathrm{~m}$ à $8,50 \mathrm{~m}$, ces travaux permettant aux navires de se croiser facilement et d'accélérer leur vitesse. En complément, pour pouvoir accueillir les navires de dimensions exceptionnelles, neuf nouvelles gares de $15 \mathrm{~m}$ de largeur supplémentaire et de $750 \mathrm{~m}$ de long, presque toutes situées entre Port-Saïd et le lac Timsah, ont été aménagées ${ }^{8}$. Le chenal a par ailleurs été éclairé par des balises au gaz comprimé pour permettre la navigation de nuit ${ }^{9}$. En complément, des projecteurs électriques ont été installés sur les navires.

Le travail d'actualisation du plan en relief a de nouveau été confié à Charles Muret. Il a ainsi fait figurer sur le plan-relief :

- le canal d'eau douce Abbasieh, creusé de 1887 à 1893 entre Ismaïlia et Port-Saïd pour amener l'eau du Nil dans cette ville ;

- la ligne du tramway à vapeur, reliant Ismaïlia à Port-Saïd, ouverte au public en 1893 ;

- les lignes télégraphiques et téléphoniques de la Compagnie du canal, figurées par deux réseaux de fils bruns et de fils noirs ;

- les surélargissements du canal creusés depuis 1896 pour permettre le croisement des navires de grande largeur. Dans le même temps, la teinte

7. Compagnie universelle du canal maritime de Suez, Exposition universelle internationale Paris 1900. Notice sur la participation de la Compagnie, Paris, Imprimerie générale Lahure, 1900, p. 26.

8. "Canal de Suez ", Revue technique de l'Exposition universelle de 1900, Texte 7, 4 partie, Génie civil, t. II, 1901, p. 323-324.

9. Stanislas Meunier, "Nouvelles du canal de Suez ", Académie des sciences. Séance du $1^{\text {er }}$ avril 1889, La Nature, 1889, $17^{\text {` }}$ année, premier semestre, p. 303. 
bleue des eaux ayant $9 \mathrm{~m}$ ou plus de profondeur a été renforcée sur la maquette ;

- l'emplacement des gares pour les navires tout le long du canal ;

- le tracé des villes a aussi été modifié, figurant leur agrandissement, le tracé des îlots encore à bâtir et les limites des terrains concédés à la Compagnie ;

- Les plantations effectuées depuis 1870 aux abords des villes pour l'agrément des habitants ont été représentées, comme celles qui ont été faites depuis 1896, en bordure du canal maritime et du canal Abbasieh, pour la protection des berges et l'arrêt des sables soulevés par le vent ;

- cent quarante petites pierres brillantes, blanches, rouges ou vertes, ont été mises en place sur la maquette, le long du canal, pour figurer les phares, feux et bouées lumineuses aménagés depuis 1885 pour faciliter la navigation de nuit ;

- de petites plaques métalliques montées sur tiges verticales et portant écrites sur les deux faces, les désignations des localités, gares, lacs, montagnes, etc. ont été ajoutées. Sur les soixante qui avaient été commandées à Charles Muret, seules quatre subsistent aujourd'hui.

À l'occasion de cette mise à jour, Muret signale à la Compagnie universelle du canal de Suez que certaines inexactitudes du plan du canal réalisé en 1878 étaient voulues ou forcées, en raison de la différence des échelles, en largeur et en longueur, et de l'agrandissement des villes. Selon lui, les seuls points à corriger correspondaient à des erreurs de positions, telles que les gares changées de rives ou le chenal déplacé ${ }^{10}$. D’autres distorsions par rapport à la réalité sont aussi à signaler. En janvier 1900 la Compagnie faisait remarquer à Muret la disproportion des surfaces occupées sur le plan par le lac Timsah et les lacs Amer. Pour Muret, il n'était pas possible d'agrandir le lac Amer, au risque de détériorer le papier mâché agglutiné utilisé pour la figuration des terres. Pour réduire l'effet d'inexactitude, le topographe propose de diminuer la surface occupée par Ismaïlia du côté de la ville égyptienne ${ }^{11}$.

10. Exposition universelle de 1900. Plans-reliefs du canal et de Port-Saïd. 20 juin 1899. ANMT, fonds "Compagnie universelle du canal maritime de Suez, puis Compagnie financière de Suez ", Carton 19950604153 / 93, Plans en relief du canal et des villes (1889-1908).

11. Sous-pochette : Exposition universelle 1900. Notes du 20 juin 1899 et du 30 janvier 1900. ANMT, fonds " Compagnie universelle du canal maritime de Suez, puis Compagnie financière de Suez », Carton 19950604153 / 93, Plans en relief du canal et des villes (1889-1908). 
Conservé et exposé dans la salle des titres de la Compagnie universelle du canal de Suez entre deux expositions universelles, le plan-relief réalisé par Charles Muret a été donné à la galerie des Plans-reliefs en 1910. Il constitue aujourd'hui un précieux témoignage de l'état du canal de Suez et des villes et équipements aménagés à ses abords à la fin du XIX ${ }^{\mathrm{e}}$ siècle, que la double exposition organisée par l'Institut du monde arabe et le musée d'Histoire de Marseille a contribué à révéler.

\section{L'autrice}

Isabelle Warmoes est ingénieur d'études au Musée des Plans-reliefs, adjointe au directeur. Elle est membre de la commission « Histoire » du Comité français de cartographie. Ses recherches sont consacrées à l'histoire de la fortification et de l'architecture militaire ( $\mathrm{xv} \mathrm{l}^{\mathrm{e}}$-xIx ${ }^{\mathrm{e}}$ siècles), des ingénieurs militaires, de la cartographie, des plans-reliefs. Elle a notamment publié, avec Émilie d'Orgeix, Les savoirs de l'ingénieur militaire. Manuels, cours et cahiers d'exercices (1751-1914) », Actes des $5^{\text {es }}$ journées d'études du Musée des plans-reliefs, Ministère de la Culture et de la Communication, Direction des patrimoines, Musée des Plans-reliefs, 2013 et Atlas militaires manuscrits (XVII ${ }^{e}$-XVIII siècles). Villes et territoires des ingénieurs $d u$ roi, Paris, Bnf Éditions/Ministère des Armées, 2017.Contact : isabelle.warmoes@ culture.gouv.fr 